\author{
Pascual Sanchez-Juan \\ Raquel Sánchez-Valle \\ Alison Green \\ Anna Ladogana \\ Natividad Cuadrado-Corrales \\ Eva Mitrová \\ Katharina Stoeck \\ Theodoros Sklaviadis \\ Jerzy Kulczycki \\ Klaus Hess \\ Anna Krasnianski \\ Michele Equestre \\ Danka Slivarichová \\ Albert Saiz \\ Miguel Calero \\ Maurizio Pocchiari \\ Richard Knight \\ Cornelia M. van Duijn \\ Inga Zerr
}

Received: 17 June 2006

Received in revised form: 21 August 2006

Accepted: 17 September 2006

Published online: 25 March 2007

Pascual Sanchez-Juan and Raquel SánchezValle contributed equally to the manuscript.

P. Sanchez-Juan, MD · C. M. van Duijn, PhD Dept. of Epidemiology and Biostatistics

Erasmus University Medical Center

PO Box 1738

3000 DR Rotterdam

The Netherlands

R. Sánchez-Valle, MD $(\varangle) \cdot$ A. Saiz, MD

Dept. of Neurology

Hospital Clínic, Institut d'Investigació

Biomèdica August Pi i Sunyer

Villarroel 170

08036 Barcelona, Spain

Tel.: +34-932275414

Fax: +34-932275783

E-Mail: 31799rsd@comb.es

A. Green, PhD $\cdot$ R. Knight, FRCP(E)

National CJD Surveillance Unit

The University of Edinburgh

EH4 2XU Edinburgh, UK

\title{
Influence of timing on CSF tests value for Creutzfeldt-Jakob disease diagnosis
}

Abstract Background The analysis of markers in the cerebrospinal fluid (CSF) is useful in the diagnosis of sporadic Creutzfeldt-Jakob disease (sCJD). However, the time at which the study of these markers is most sensitive remains controversal. Objective To assess the influence of time of sampling on the value of CSF tests in the diagnosis of sCJD. Method In the framework of a multinational European study, we studied the results of 14-3-3, S100b, neurone specific enolase (NSE) and tau protein in $833 \mathrm{CSF}$ samples

A. Ladogana, MD - M. Equestre, MD M. Pocchiari, $\mathrm{PhD}$

Dept. of Cell Biology and Neurosciences Instituto Superiore di Sanità

Viale Regina Elena 299

00161 Rome, Italy

N. Cuadrado-Corrales, $\mathrm{PhD}$

M. Calero, $\mathrm{PhD}$

Instituto de Salud Carlos III

Sinesio Delgado 6

28029 Madrid, Spain

E. Mitrová, MD · D. Slivarichová, MSc Institute of Preventive and Clinical Medicine

Limbová str. 14

83301 Bratislava, Slovakia from sCJD patients at different stages of disease and in 66 sequentially repeated lumbar punctures (LP). Results 14-3-3 and tau protein tended to increase in sensitivity from onset $(88 \%$, $81 \%)$ to the advanced stage $(91 \%$, $90 \%)$. This was significant only in the methionine-valine (MV) heterozygous group of patients at codon 129. The absolute levels of S100b $(\mathrm{p}<0.05)$, NSE and tau protein increased in the last stage of disease. High levels of tau protein, NSE and S100b were associated with shorter survival times $(p<0.01)$. Sixty-six sCJD patients underwent repeated LP. These sCJD patients were younger, had longer disease durations and were more frequently $\mathrm{MV}$ at codon $129(\mathrm{p}<0.001)$ than the whole group. 14-3-3 sensitivity increased from $64 \%$ to $82 \%$ in the second LP $(p=0.025)$ and $88 \%$ sCJD patients had at least one positive result. Conclusions Sensitivity and absolute levels of CJD markers increased with disease progression and were modulated by the codon 129 genotype. Early negative results should be inter- 


\section{K. Stoeck, MD}

National Reference Center for Human Prion Diseases (NRPE)

Institute of Neuropathology

Schmelzbergstr.12

8091 Zurich, Switzerland

T. Sklaviadis, $\mathrm{PhD}$

Laboratory of Pharmacology

Dept. of Pharmaceutical Sciences

School of Health Sciences

Aristotle University of Thessaloniki

54124 Thessaloniki, Greece

J. Kulczycki, PhD

I-st Neurological Department

Institute of Psychiatry and Neurology

Sobieskiego 9

02-957 Warsaw, Poland preted with caution, especially in young patients or those who are MV at codon 129.

\section{K. Hess, MD}

Dept. of Neurology

University Hospital Zurich

Frauenklinikstr. 26

8091 Zurich, Switzerland

A. Krasnianski $\cdot$ I. Zerr, MD

Dept. of Neurology

National TSE reference center

Georg-August-University Göttingen

Robert-Koch-Str. 40

37075 Göttingen, Germany
Key words Creutzfeldt-Jakob disease prion $14-3-3$.

tau $\cdot \mathrm{S} 100 \mathrm{~b} \cdot \mathrm{NSE} \cdot$

biological markers . repeated lumbar punctures . prognosis

\section{Introduction}

Creutzfeldt-Jakob disease (CJD) is a rapidly progressive neurodegenerative disease. Presently, the definite diagnosis of CJD requires neuropathological confirmation. Cerebrospinal fluid (CSF) analysis plays an important role in the diagnosis of CJD. After the WHO consultation in 1998, a positive 14-33 result is considered as a criterion of probable sporadic CJD (sCJD) [12]. Different studies have shown that in the appropriate clinical circumstances a positive 14-3-3 is highly sensitive and specific for the diagnosis of sCJD [14]. However, the question remains open as to whether 14-3-3 is more sensitive in the initial or late stages of disease. Increased levels of other brain-derived proteins, such as microtubule-associated tau protein [8], S100b [4] or neurone specific enolase (NSE) [13] have also been reported to be elevated in CSF in CJD. The published data about how the levels of these proteins are modified during the course of disease are contradictory $[1-3,5-7,10,11]$. In addition, the time point during the disease, when biochemical markers are expected to have the highest sensitivity, is not welldefined. These topics have not been studied systematically, especially because data from sequential CSF analyses are rarely available.

In the framework of an European Community supported multinational study on CJD markers, we performed an analysis of the results obtained in different stages of the disease and in repeated lumbar punctures (LP) to assess the influence of time of sampling on the value of CSF tests in the diagnosis of sCJD.

\section{Methods}

\section{Study overview}

This study was conducted in the framework of a European Community supported multinational study (Early clinical diagnosis of human spongiform encephalopathies by analysis of biological fluids (CJDmarkers), (QLG3-CT-2002-81606 Coordinator: I. Zerr). All participant countries belong to the EUROCJD Surveillance network with comparable surveillance methods. CSF samples were sent to the individual laboratories for 14-3-3 analysis in the participating countries between 1998-2003 and clinical and pathological data were collected. CSF was obtained by LP and the analyses were conducted in each laboratory following standard protocols [9]. An analysis of test validity and comparability between laboratories revealed high agreement between laboratories [9]. Previously published cut-off values were used for the quantitative markers $[8,13]$.

In all countries, clinical and neuropathological data from patients with clinical suspicion of CJD or related disorders were collected. The diagnoses of CJD and various CJD subtypes were made according to the established criteria in all participating centers. Core clinical data such as age at onset, gender, disease duration, time point of the LP, codon 129 genotype, $\mathrm{PrP}^{\mathrm{res}}$ type and final clinical and neuropathological diagnoses were centralized by each center. A database was set up, which included detailed data on the CSF markers and patients' characteristics. The study was performed in accordance with the ethical standards laid down in the 1964 Declaration of Helsinki.

\section{Statistical analyses}

Since the clinical duration within SCJD patients varies widely depending upon several variables (e.g., age at onset, gender, codon 129 polymorphism of the PRNP gene, and $\operatorname{PrP}^{\text {res }}$ type), the time interval between LP and disease onset is not an appropriate estimate of the stage of disease when the LP was performed. To overcome this problem, we divided the time of LP to disease onset in each patient by the total duration of the disease. Thus, we classified patients in three categories according to whether they 
underwent LP in the first (time of LP to disease onset/ total duration of the disease $<0.33)$, second $(0.33-0.66)$ or third $(>0.66)$ stage of the disease. We analyzed the sensitivity of each marker at different stages of the disease. The differences in sensitivities across strata were analyzed using a logistic regression model adjusting for possible confounders and covariates. We also performed a stratified analysis by codon 129 PRNP genotype. In a similar way we assessed the absolute levels of quantitative markers across the different stages of the disease. The absolute levels of S100b obtained with different methods were analysed separately.

In order to evaluate the correlation between tau protein, S100b or NSE levels and disease duration we used the non-parametric correlation coefficient Spearman's Rho. Differences in CSF tau protein levels between patients with disease duration above or below the median (5 months) were compared using the MannWitney test. For the second part of the study, we selected patients who underwent more than one LP during the course of disease and then compared the sensitivity and evolution of each of the CJD markers. Differences in sensitivities were assessed using chi ${ }^{2}$ test.

\section{Results}

One thousand five hundred and fifty two samples from sCJD patients were studied. The time of LP and disease onset was available in 833 patients and the median time from onset to LP was 3 months (range 0.1-84.5). The LP was performed during the first stage of the disease in $16 \%(\mathrm{n}=137)$, during the second stage in $38 \%(\mathrm{n}=316)$, and in the final stage in $46 \%$ $(\mathrm{n}=380)$. The median time from disease onset to LP in patients who underwent the LP in the first stage of the disease was 1.9 months (range 0.1-10.2); 2.3 months (0.2-23.6) in patients that underwent the LP in the second stage of the disease and 3.9 months (0.7-84.5) for those in the third stage. The data on test sensitivity for each marker stratified by the disease stage are given in Table 1 . The sensitivity of 14-3-3 tended to increase from the first $(88 \%)$ to the third stage of the disease (91\%), although these differences were not statistically significant. When stratifying by codon 129 of PRNP, this trend became significant in the group of MV patients ( $\mathrm{p}=0.01)$.

Similar results were obtained for tau protein. There were not significant differences in the sensitivity of tau or in the median level of tau across the three disease stages (sensitivity of $81 \%$ in the first stage and $90.5 \%$ in the third stage, $\mathrm{n}=342$ ). However, when stratifying by codon 129 , again the trend toward a higher sensitivity through the disease stages became significant in the group of MV patients $(p=0.048)$ (Table 1). There was a trend of a gradual increase of tau levels in the MV patients, with the highest levels in the last stage (median $1202 \mathrm{pg} / \mathrm{mL}$ in the first stage, $1909 \mathrm{pg} / \mathrm{mL}$ in the second and 2601 in the third) and in VV patients (median $5949 \mathrm{pg} / \mathrm{mL}$ in the second and 10422 in the third stage of the disease).

S100b levels were analysed in 297 samples, 37 in the first stage (12\%), 112 in the second (38\%), 148 in the third (50\%.). The disease stage clearly influenced the $S 100 \mathrm{~b}$ sensitivity with a significant increase $(p=0.01)$ in the latest stage (Table 1$)$. The absolute levels of S100b (Byk-Sangtec kit) also increased across the disease $(\mathrm{p}<0.05)$.

NSE levels were assessed in 217 samples, and those obtained in the last stage gave a significantly higher sensitivity (83\%) when compared to the second stage $(67 \%, p=0.01)$.

Levels of tau protein, S100b and NSE in CSF were negatively correlated with disease duration (nonparametric correlation coefficient Spearman's Rho = tau innogenetics $-20 \%(\mathrm{p}<0.001)$, S100b Byk-Sangtec $-17 \% \quad(\mathrm{p}=0.002), \quad \mathrm{S} 100 \mathrm{~b}$ in house $-18 \%$ $(\mathrm{p}=0.01), \quad$ NSE Hoffman-la Roche $-21 \%$ $(p=0.001))$. Patients with a median disease duration of 5 months or less had a median tau protein level of $6407 \mathrm{pg} / \mathrm{mL}$ (75-3623), whilst patients with a disease duration of longer than 5 months had a median tau protein level of $4411 \mathrm{pg} / \mathrm{mL}$ (75-47150), (Mann-Witney test $\mathrm{p}<0.001$ ).

Sixty-six out of 1552 (4\%) sCJD patients underwent more than one LP (at least two). The median time lapse between the LPs was 6 weeks. Forty-two (64\%) of these cases were positive for 14-3-3 in the first LP (Figure 1). This value was different from the sensitivity in the whole group $(85 \%, \mathrm{p}<0.01)$. Fiftyfour (82\%) of sCJD cases had a positive 14-3-3 in the second sample $(\mathrm{p}=0.025)$. Thirty-eight $(57 \%)$ sCJD patients had a positive 14-3-3 in both samples, and 58 (88\%) patients had at least one positive 14-3-3. Twenty sCJD patients $(30 \%)$ changed their result for 14-3-3 in the second LP. Sixty-six percent of false negative sCJD became positive but $9.5 \%$ of those positive for 14-3-3 became negative in the second LP.

The sensitivity of the other markers was also significantly lower in the first LP when compared to the whole group of sCJD $(p<0.05)$. However, the sensitivity of these markers increased in the second sample when compared to the first, although not significantly. Eighty-one percent of sCJD cases had positive tau protein levels in one of the two LPs, but only $61 \%$ of cases had positive levels in both of them (Figure 1). With respect to $\mathrm{S} 100 \mathrm{~b}$ and NSE, $66 \%$ and $70 \%$ of sCJD patients had positive results in one of the two LPs and $53 \%$ and $33 \%$ in both of them, respectively.

We also found that the absolute levels of all three quantitative markers tended to increase in the second LP. This tendency was significant for S100b (median levels: $4.5 \mathrm{ng} / \mathrm{ml}(1-112)$ in the first LP, median levels: $7 \mathrm{ng} / \mathrm{ml}(1-38)$ in the second; $\mathrm{p}=0.027$ ) (Figure 2). In 39 out of $52(75 \%)$ samples the levels of tau protein were higher in the second LP than in the first LP. NSE levels were higher in the second LP when compared to the first in 32 out of $46(70 \%)$ samples, and S100b 
Table 1 Sensitivity of markers in sporadic Creutzfeldt-Jakob disease at different disease stages, in the whole group and stratified by codon 129 PRNP genotype.

\begin{tabular}{|c|c|c|c|c|c|c|c|}
\hline & first stage $\dagger$ & $\mathrm{N}$ & second stage $\dagger$ & $\mathrm{N}$ & third stage $\dagger$ & $\mathrm{n}$ & $p$ for trend* \\
\hline Positive $14-3-3$ & 87.6 & $120 / 137$ & 88.0 & $278 / 316$ & 91.3 & $347 / 380$ & Ns \\
\hline $\mathrm{MM}$ & 94.0 & $79 / 84$ & 90.6 & $155 / 171$ & 93.3 & $166 / 178$ & Ns \\
\hline MV & 60.0 & $15 / 25$ & 69.8 & $37 / 53$ & 89.2 & $58 / 65$ & 0.01 \\
\hline VV & 100.0 & $6 / 6$ & 95.7 & $44 / 46$ & 92.2 & $47 / 51$ & Ns \\
\hline Positive tau & 80.9 & $38 / 47$ & 84.9 & $107 / 126$ & 90.5 & $153 / 169$ & Ns \\
\hline MM & 83.9 & $26 / 31$ & 88.2 & $60 / 68$ & 92.9 & $79 / 85$ & Ns \\
\hline MV & 42.9 & $3 / 7$ & 65.2 & $15 / 23$ & 80.6 & $25 / 31$ & 0.048 \\
\hline VV & 100.0 & $1 / 1$ & 100.0 & $17 / 17$ & 96.0 & $24 / 25$ & Ns \\
\hline Positive S100b & 73.0 & $27 / 37$ & 81.3 & $91 / 112$ & 93.9 & $139 / 148$ & 0.01 \\
\hline MM & 78.6 & $22 / 28$ & 81.4 & $48 / 59$ & 97.1 & 68/70 & 0.02 \\
\hline MV & 25.0 & $1 / 4$ & 66.7 & $10 / 15$ & 78.6 & $22 / 28$ & Ns \\
\hline VV & 0.0 & $0 / 1$ & 89.5 & $17 / 19$ & 100.0 & $23 / 23$ & Ns \\
\hline Positive NSE & 82.5 & $33 / 40$ & 67.1 & $53 / 79$ & 82.7 & $81 / 98$ & 0.01 \\
\hline MM & 86.7 & $26 / 30$ & 68.9 & $31 / 45$ & 84.9 & $45 / 53$ & 0.02 \\
\hline MV & 50.0 & $2 / 4$ & 41.7 & $5 / 12$ & 72.7 & $16 / 22$ & Ns \\
\hline VV & 0.0 & $0 / 1$ & 76.9 & $10 / 13$ & 87.5 & $14 / 16$ & Ns \\
\hline
\end{tabular}

* adjusted by country of origin, gender, disease duration, age of onset

$\dagger$ percentages

$\mathrm{MM}=$ methionine homozygous; $\mathrm{MV}=$ methionine/valine; $\mathrm{VV}=$ valine homozygous

NSE = neurone specific enolase. The cut-off value for tau was $1,300 \mathrm{pg} / \mathrm{ml}$; for S100b $4.2 \mathrm{ng} / \mathrm{ml}$, for the Byk-Sangtec ${ }^{\circledR}$ kit and $0.5 \mathrm{ng} / \mathrm{ml}$ for the, Non-commercial kit used in the United Kingdom; and $35 \mathrm{ng} / \mathrm{ml}$ for the Hoffman La Roche ${ }^{\circledR}$ kit and $25 \mathrm{ng} / \mathrm{ml}$ for Byk-Diasorin ${ }^{\circledR}$, Byk-Sangtec ${ }^{\circledR}$ and Wallac ${ }^{\circledR}$ kits for NSE.

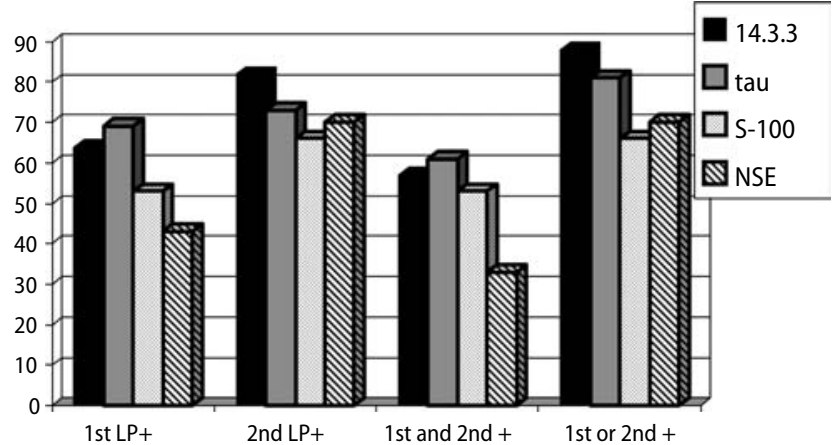

Fig. 1 Sensitivity of markers for sporadic Creutzfeldt-Jakob disease diagnosis in repeated lumbar punctures (LP) and combined results in both LP. $\mathrm{LP}=$ lumbar puncture, $+=$ positive, NSE $=$ neurone specific enolase, and $=$ both $\mathrm{LP}+$, or $=$ either $1^{\text {st }}$ or $2^{\text {nd }}+$

levels were higher in the second LP when compared to the first in 27 out of $38(71 \%)$ samples.

When we compared the characteristics of the sCJD patients who underwent repeated LP to the overall group of sCJD we found that these patients were younger at onset (mean age: 61years SD 9.8 vs. 66 years SD 9.6) and more frequently under 40 years $(6 \%$ vs. $1 \%, \mathrm{p}<0.001)$. They had a longer disease duration (mean: 12.8 months SD 8.7 vs. 7.9 months SD 7.8) and were more frequently heterozygous at codon 129 $(\mathrm{p}<0.001)$.

\section{Discussion}

Previous data about the influence of timing on the levels of CSF markers for CJD diagnosis are contra- dictory. The studies vary with respect to their methodology and often only single patients were included in the analysis. Jimi et al. [5] showed in a sequential study that the levels of CJD markers increased during the course of the disease and returned to normal or mildly elevated levels in the terminal stage. Mollenhauer et al. [7] also discussed the difficulties of CJD diagnosis in advanced cases presenting one sCJD case that became 14-3-3 negative in the final stage of the disease. On the other hand, Giraud et al. [3] reported an increase in the levels of 14-3-3 proteins during the course of the disease in a limited number of sCJD cases. Brandel et al. [2] performed a sequential study in iatrogenic CJD, and observed that $14-3-3$ was rarely detectable within the first 3 months of the disease but always positive after 7 months associated with aggravation of the disease and the occurrence of dementia. The genotype at PRNP codon 129 influenced the timing of the rise of 14-3-3 in the CSF in these cases. Kropp et al. [6] studied NSE levels in 16 sCJD patients with repeated LP. In 15 of them, NSE levels were higher in the second LP with respect to the first, but no other markers were studied and the disease duration or the PRNP genotype were not analysed. Finally, in a recent study, van Everbroeck et al. [11] reported that in $42 \mathrm{sCJD}$ patients the concentrations of 14-3-3 or tau protein were higher in the middle point of the disease (arbitrarily determined between the $25 \%$ and $85 \%$ of the total disease duration) than in the early or late stage.

In the framework of a multinational project, we studied the influence of timing on the CSF marker results in $833 \mathrm{sCJD}$ patients. As the disease duration 
Fig. 2 Levels of quantitative markers in repeated lumbar punctures. ${ }^{1}$ Differences between median levels were not significant. ${ }^{2}$ THe median S100b levels were different $(p=0.027)$. ${ }^{*}$ Byk-Sangtec kit
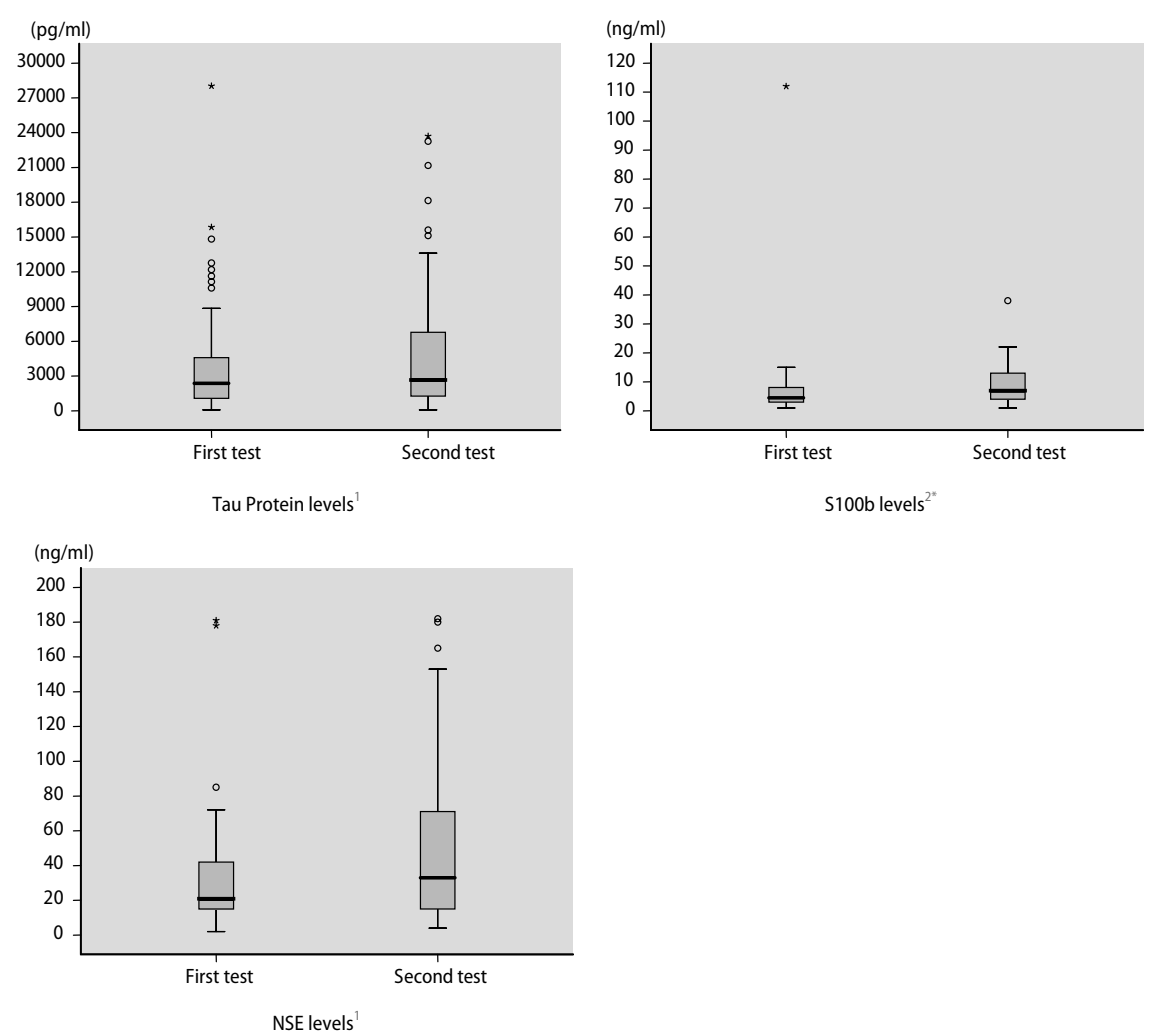

differs among CJD patients, we decided to analyse the influence of the stage of the disease, and not the absolute time to the disease onset or death, on the results of the CSF markers in order to avoid a bias towards the results in patients with shorter disease duration. On the other hand, in order to study the influence of timing on the different PRNP genotypes we also performed a stratified analysis by the PRNP genotype.

Our study showed a tendency for the sensitivity of all CSF markers to increase and for the absolute levels of the quantitative markers to rise during the course of the disease, using both the multivariate analysis and the assessment of the repeated LPs. Thus, CSF markers had the highest sensitivity at the third stage of disease. The codon 129 PRNP genotype modified this effect. The trend for a higher sensitivity of 14-3-3 and tau protein for SCJD diagnosis in the later stages of disease only became significant in the group of codon $129 \mathrm{MV}$ patients. In addition we found that higher levels of tau protein were associated with a shorter survival, similiar to the results of another study, where CSF phosporylated tau protein levels were analysed [10]. High levels of other brain-derived proteins (S100b, NSE) were also significantly associated with shorter survival. These brain-derived proteins are released into the CSF as a result of cell damage or death, and as such the levels of these markers reflect the rate of cell death are probably inversely related to disease duration. However, we should admit that we did not assess separately the sensitivity of the markers at very late stages of disease and we cannot rule out a possible final drop in sensitivity, paired to a slower rate of cell death at the final stage.

In those sCJD patients who had more than one LP performed, we found that the sensitivity of all CSF markers increased in the second LP when compared to the first LP. In more than $70 \%$ of all sCJD cases tested the absolute levels of the three quantitative markers also increased in the second LP. Sporadic CJD patients who underwent a second LP differed from the whole group, in that they were younger, had a longer disease duration and were more frequently heterozygous for PRNP codon 129 when compared to the whole group. These characteristics made them a more difficult group to diagnose and may explain why a second LP was performed. In this group of atypical sCJD patients a second LP increased the 14-3-3 sensitivity by $18 \%$.

In conclusion, in our study we found that the sensitivity of the CSF markers for sCJD diagnosis increased during disease progression and that this was modulated by the codon 129 genotype. High levels of CSF brain-derived proteins were associated with a shorter survival. A negative CSF 14-3-3 result 
in the early stages should be interpreted with caution, especially in MV patients. Although performing a second LP is not usual clinical practice for the diagnosis of CJD, it may be useful to perform a second LP, 4-6 weeks after the initial one, in young patients, those with long clinical course or MV genotype.

Acknowledgements We thank all physicians in the participating countries for sending us the cerebrospinal fluid and blood samples and for providing pertinent clinical and neuropathological data on those patients. We thank Jolanthe Zellner for her coordinative work and Esther Croes for database management at the initial stages of the project. The collaborative study was funded by grants from the European Commission (EC) (QLG3-CT-2002-81606 to I. Zerr).

The national studies were supported in Greece by the Greek Ministry of Health, through KEEL (Center for Control of Infectious
Diseases), in Italy by the Ministry of Health and the Instituto Superiore di Sanità, in the Netherlands by the Dutch Ministry of Health, Welfare and Sports, in Slovakia by Ministry of Health and European Commission (SEEC-CJD project), in Spain by a grant from the Ministerio de Ciencia y Tecnología (MCyT EET 2001/ 2216) and Spanish Ministerio de Sanidad y Consumo, grant number DGVI 1312/04-1, in Switzerland by Bundesamt für Gesundheit, Bern (NRPE-BAG contracts $n^{\circ}$ 03.001297and $n^{\circ}$ 04.002363), in United Kingdom by the Department of Health and the Scottish Home Office Department of Health and in Germany by the Bundesministerium für Gesundheit und Soziale Sicherung (BMGS) (GZ: 325-4471-02/15) and by the Bundesministerium für Bildung und Forschung (BMBF) (KZ: 0312720 and 01GI0301 to I.Z). Raquel Sanchez-Valle is a recipient of a post-residency grant from Instituto de Salud Carlos III, Madrid, Spain. Pascual Sanchez-Juan was supported by the postMIR grant Wenceslao Lopez Albo from the IFIMAV Institute of the Fundación Pública Marqués de Valdecilla.

\section{References}

1. Beaudry P, Cohen P, Brandel JP, et al. (1999) 14-3-3 protein, neuron-specific enolase, and S100b protein in cerebrospinal fluid of patients with Creutzfeldt-Jakob disease. Dement Geriatr Cogn Disord 10(1):40-6

2. Brandel JP, Peoc'h K, Beaudry P, et al. (2001) 14-3-3 protein cerebrospinal fluid detection in human growth hormone-treated Creutzfeldt-Jakob disease patients. Ann Neurol 49(2):257-60

3. Giraud P, Biacabe A-G, Chazot G, et al. (2002) Increased detection of 14-3-3 protein in Cerebrospinal fluid in Sporadic Creutzfeldt-Jakob disease during the disease course. Eur Neurol 48:218221

4. Green AJE, Keir G, Thompson EJ (1997) A specific and sensitive ELISA for measuring S100b in the cerebrospinal fluid. J Immmunol Methods 205:35-41
5. Jimi T, Wakayama Y, Shibuya S, et al. (1992) High levels of nervous systemspecific proteins in cerebrospinal fluid in patients with early stage CreutzfeldtJakob disease. Clin Chim Acta 211:3746

6. Kropp S, Zerr I, Schulz-Schaeffer WJ, et al. (1999) Increase of neuron-specific enolase in patients with CreutzfeldtJakob disease. Neurosc Lett 261(12):124-6

7. Mollenhauer B, Serafin S, Zerr I, et al. (2003) Diagnostic problems during late course in Creutzfeldt-Jakob disease. J Neurol 250:629-630

8. Otto M, Wiltfang J, Cepek L, et al. (2002) Tau protein and 14-3-3 protein in the differential diagnosis of Creutzfeldt-Jakob disease. Neurology 58:192197

9. Sánchez-Juan P, Green A, Ladogana A, et al. (2006) CSF tests in the differential diagnosis of Creutzfeldt-Jakob disease. Neurology 67(4):637-643
10. Van Everbroeck B, Green AJ, Vanmechelen E, et al. (2002) Phosphorylated tau in cerebrospinal fluid as a marker for Creutzfeldt-Jakob disease. J Neurol Neurosurg Psychiatr 73(1):79-81

11. Van Everbroeck B, Quolin S, Boons J, et al. (2003) A prospective study of CSF markers in 250 patients with possible Creutzfeldt-Jakob disease. J Neurol Neurosurg Psychiatry 74:1210-1214

12. WHO. Human transmissible spongiform encephalopathies (1998) Wkly Epidemiol Rec 73:361-72

13. Zerr I, Bodemer $M$, Räcker $S$, et al. (1995) Cerebrospinal fluid concentration of neuron-specific enolase in diagnosis of Creutzfeldt-Jakob disease. Lancet 345:1609-1610

14. Zerr I, Pocchiari M, Collins S, et al. (2000) Analysis of EEG and CSF 14-3-3 proteins as aids to the diagnosis of Creutzfeldt-Jakob disease. Neurology 55:811-815 